\title{
Investigation of Laser-Mode Anticompetition in Semiconductor Lasers
}

\author{
Chi-Chia Huang, Yi-Shin Su, and Ching-Fuh Lin, Senior Member, IEEE
}

\begin{abstract}
Influential factors of laser-mode anticompetition are investigated in dual-wavelength semiconductor laser. Experiment shows that with increasing wavelength, separation, or decreasing initial power of the long-wavelength mode (LWM), the slope of the anticompetition curve and the maximum power increment of the LWM increase. Under fixed wavelength separation, anticompetition can exist only when the power of the short-wavelength mode (SWM) is below a certain level. In addition, the wavelength position has an effect on anticompetition. Different injection current also results in different behaviors of anticompetition. Anticompetition can only be observed with varying the power of the SWM.
\end{abstract}

Index Terms-Anticompetition, broad-band, competition, multiple quantum well (MQW), semiconductor laser.

\section{INTRODUCTION}

C OMPETITION of laser modes is a well-known phenomenon in laser systems [1]. In a multimode laser system, different modes compete for the available population inversion in the laser system. Since the total gain of a laser system is constant under a fixed pumping level, oscillation in one mode will generally reduce the gain available for another mode, and in some situations may suppress the other mode completely [2]. Therefore, in a dual-wavelength laser system, increasing the intensity of one mode decreases the intensity of another mode. The behavior of competition can lead to bistability of the dual-wavelength laser system under strong coupling condition [2], which had potential applications such as wavelength switching [3] and electro-optical logic operations [4]. Competition can also lead to tristability [5], which should extend the potential applications and advantages of the two-mode laser systems [6], [7]. Mode competition can also result in chaotic behaviors [8], [9], which were found useful for optical switching, optical logic, data encryption [10], [11], etc. In addition, competition dynamics is an important phenomenon in injection locking [12], mode locking [13]-[15], and cross gain modulation in optical amplifiers [16]. Soon after the invention of lasers, competition of laser modes has been observed for decades and has been thought to be inevitable. However, Lin et al. has discovered that the increase of the oscillation intensity in one lasing mode can, at some situation, enhance the intensity of another mode [17]. This behavior

Manuscript received June 10, 2004; revised August 27, 2004. This work is supported in part by the National Science Council, Taipei, Taiwan, R.O.C., under Contract 92-2215-E-002-012 and Contract NSC93-2112-M-002-032.

C.-C. Huang, Y.-S. Su, and C.-F. Lin are with the Graduate Institute of Electro-Optical Engineering and the Department of Electrical Engineering, National Taiwan University, Taipei, Taiwan 106, R.O.C. (e-mail: cflin@cc.ee.ntu.edu.tw).

C.-F. Lin is with Graduate Institute of Electronics Engineering, National Taiwan University, Taipei, Taiwan 106, R.O.C.

Digital Object Identifier 10.1109/JQE.2004.838168 is opposite to the competition and is called mode anticompetition.

Anticompetition exists when the laser modes have their wavelengths widely separated [17]. Theoretical analysis in [17] shows that anticompetition is due to the physics similar to optical pumping. In our recent experiment, we have further discovered that the minimum wavelength separation at which anticompetition exists is about $60 \mathrm{~nm}$, which is much smaller than the $138 \mathrm{~nm}$ demonstrated in [17]. In addition to the wavelength separation, we have discovered that the power of each mode can also affect anticompetition. Moreover, different wavelength positions of the oscillating modes can lead to different behavior of anticompetition due to the quantum-well (QW) structure of the laser gain medium. The level of the injection current also has influences on the anticompetition. Another important phenomenon is that anticompetition cannot be observed with varying the power of the long-wavelength mode (LWM). That is, the power of the short-wavelength mode (SWM) always decreases when we increase the power of the LWM.

The investigation and discussion of the laser-mode anticompetition in this paper is organized in the following order. After this section of introduction, the experimental setup and the characteristics of the laser gain medium will be given in Section II. Then, influential factors of anticompetition will be investigated in Section III. These factors include: 1) the wavelength separation; 2) the power of the SWM; 3) the power of the LWM; 4) the wavelength position; and 5) the injection current. In Section IV, we will investigate why anticompetition cannot be observed with varying the power of the LWM. Conclusions are given in Section V.

\section{EXPERIMENTAL SETUP AND CHARACTERISTICS OF THE GAIN MEDIUM}

The experimental setup is similar to that described in [17] except that one more variable neutral-density (ND) filter is used here. Two variable ND filters are now placed in front of the two mirrors that are used for separate alignment of optical paths at two wavelengths, as shown in Fig. 1. The use of two variable ND filters greatly helps us control the intensity of both modes at the same time. To facilitate the tuning of wavelength separation, the double-slit has a $\mathrm{V}$ shape, with the long-wavelength side vertically straight and the short-wavelength side tilted at an angle of $30^{\circ}$ from the vertical line. Thus, by moving the double-slit up and down, we can easily change the wavelength separation.

The laser gain medium is a semiconductor optical amplifier (SOA) with nonidentical multiple quantum-well (MQW) structure. The QW structure of the SOA is the same as sample A 


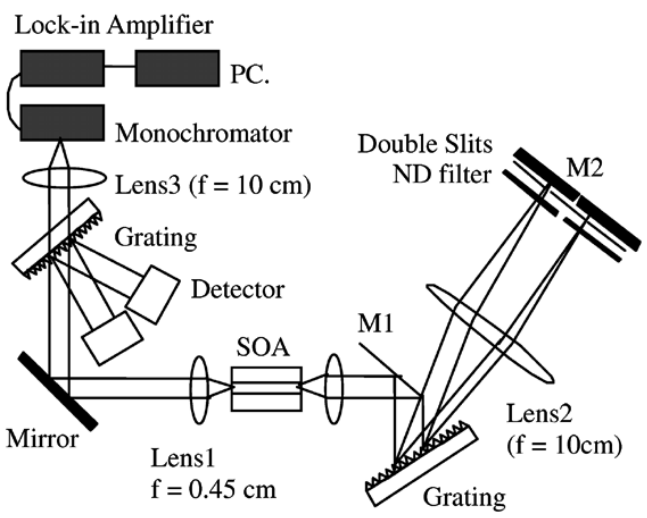

Fig. 1. Experimental setup.

in [18] and has very broad-band characteristics. The length of the device is $\sim 300 \mu \mathrm{m}$. The semiconductor laser using this QW structure for gain medium can be tuned from 1300 to $1540 \mathrm{~nm}$ under single wavelength operation [19]. However, the operation current was as large as several hundreds of milliamperes since bent waveguide was used. Because the LWM is difficult to oscillate under high injection current due to temperature effect [20], bent waveguide is not desirable. Therefore, in our experiment where the laser has to be operated under dual modes, we use SOA with straight waveguide instead [21]. It is actually a Fabry-Perot laser diode (LD) with the threshold current of $143 \mathrm{~mA}$. Most of the following experiments of anticompetition are performed at the operation current $146 \mathrm{~mA}$. Under this operation current, the Fabry-Perot mode of the LD has negligible influences on the dual-wavelength oscillation. Using this setup, we can obtain simultaneous two-mode oscillation with spectral separation tunable from a few nanometers to $191 \mathrm{~nm}$ at $146-\mathrm{mA}$ injection current and at $22.7^{\circ} \mathrm{C}$.

The output light was first measured using the monochromator to identify the wavelengths of the two lasing wavelengths. Then a grating was inserted in the output light path to separate the two beams at different wavelengths. The grating efficiency at each wavelength has been calibrated, so the output power of light at each wavelength before entering this grating could be obtained. On the other hand, the optical components such as collimators, lens, grating, etc., used in the cavity are also wavelength dependent. However, when the two wavelengths are selected by the slits, their properties are fixed at the two chosen wavelengths. The output power of the laser modes is then controlled only by the variable ND filters. In our investigation of the anticompetition, the exact measurement of the wavelength dependence of the optical components in the cavity is not necessary. Therefore, their losses as a function of the wavelength were not particularly measured.

\section{EXPERIMENTAL RESULT AND DISCUSSION-INFLUENTIAL FACTORS OF ANTICOMPETITION}

For easy description, the variable ND filter used for controlling the power of the SWM and the LWM is called the shortwavelength ND filter and the long-wavelength ND filter, respectively. The following steps are performed to obtain our experimental result. First, the loss of the short-wavelength ND filter is elevated to a value such that the SWM ceases oscillation.
Second, the long-wavelength ND filter is tuned to obtain the LWM power of about $0.07 \mathrm{~mW}$, which is called the initial LWM power. Afterwards, the loss of the long-wavelength ND filter is not changed until the end of the measurement. Finally, we gradually reduce the loss of the short-wavelength ND filter, so the SWM power gradually increases. Each time when we reduce the loss of the short-wavelength ND filter to a certain value, we record the powers of both modes. This step is repeated until the loss of the short-wavelength ND filter is reduced to zero or the LWM is completely suppressed by the SWM. Then, those measured powers are plotted on the phase plane with the LWM and SWM power as the vertical and horizontal axes, respectively. A series of curves are obtained on the phase plane.

At very small wavelength separation, the gradient of the curve is always negative, which is similar to traditional mode competition. However, at large wavelength separation, the curve is very different and can be divided into two sections. One section has negative gradient for large SWM power and is called competition curve. The other section has positive gradient for small SWM power and is called anticompetition curve. The positive gradient indicates that both the LWM and SWM powers increase at the same time, which is the characteristics of anticompetition [17]. The LWM power at the intersection of the competition and the anticompetition curves is the maximum available LWM power due to anticompetition under fixed initial LWM power. The maximum LWM power increment as a result of anticompetition is equal to the maximum LWM power minus the initial LWM power. The SWM power from zero to the corresponding power of the turning point between the competition and the anticompetition is called the existing range of anticompetition.

The curve obtained above is a curve with an initial LWM power. To obtain another curve with different initial LWM power, we only need to modify the second step described above. That is, we vary the long-wavelength ND filter to obtain another value of the LWM power. The remaining steps are then performed to obtain the curve with the new initial LWM power. Curves of different anticompetition can also be obtained by varying other influential factors. Those influential factors on anticompetition will be described in the following.

\section{A. Different Wavelength Separation}

Wavelength separation is an important factor for the occurrence of anticompetition. The experiment is as follows. First, we position the SWM and LWM at about 1360 and $1528 \mathrm{~nm}$, respectively. Then, we move the $\mathrm{V}$-shape double-slit vertically to change the spectral separation. In this way, the SWM is approximately fixed at $1360 \mathrm{~nm}$, while the LWM moves toward the short-wavelength side. The experimental results for wavelength separations $170,150,130,60$, and $30 \mathrm{~nm}$ are shown in Figs. 2(a)-(e), respectively. At 30-nm separation shown in Fig. 2(e), only competition can be observed. However, at 60-nm separation shown in Fig. 2(d), anticompetition appears. For wavelength larger than $60 \mathrm{~nm}$, anticompetition can be always observed. In addition, the gradient of the anticompetition curve increases with increasing wavelength separation. Furthermore, with fixed initial LWM power, the maximum LWM power increment also increases when we increase the wavelength separation. For example, at 150-nm separation, anticompetition 


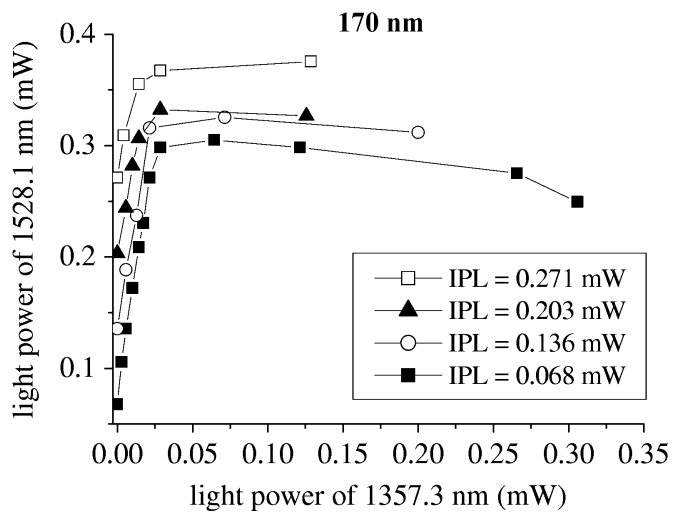

(a)

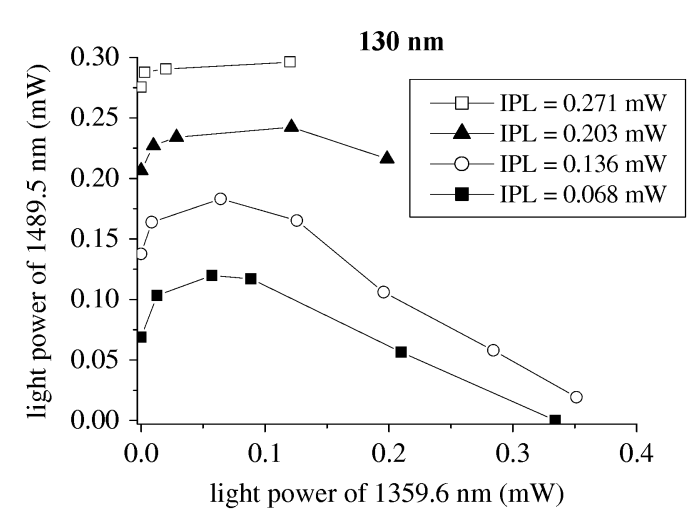

(c)

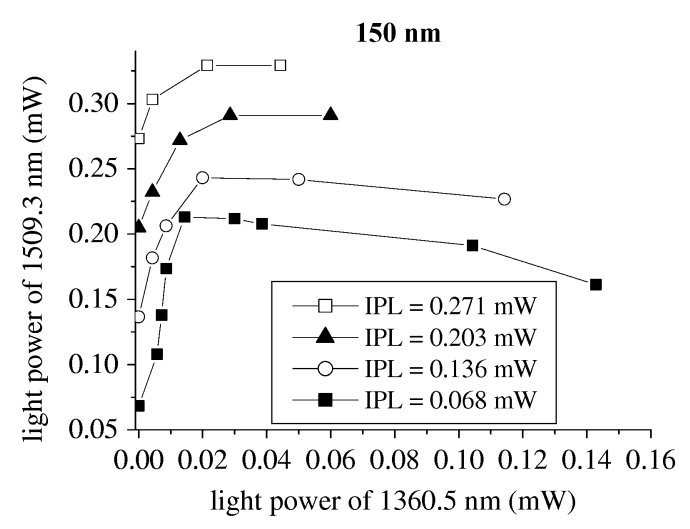

(b)

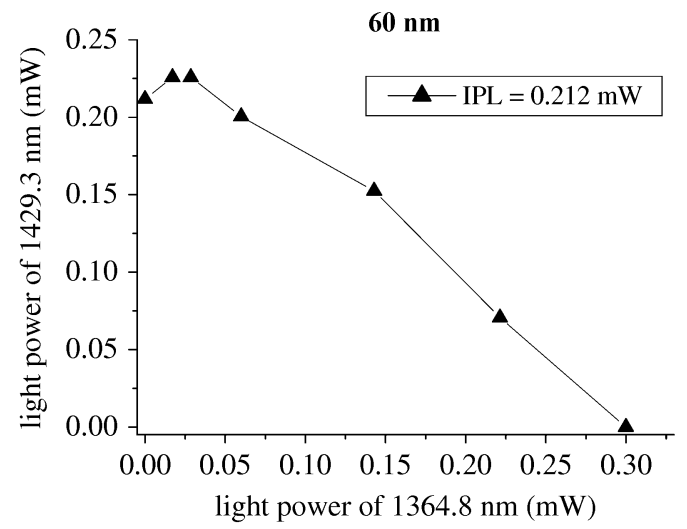

(d)

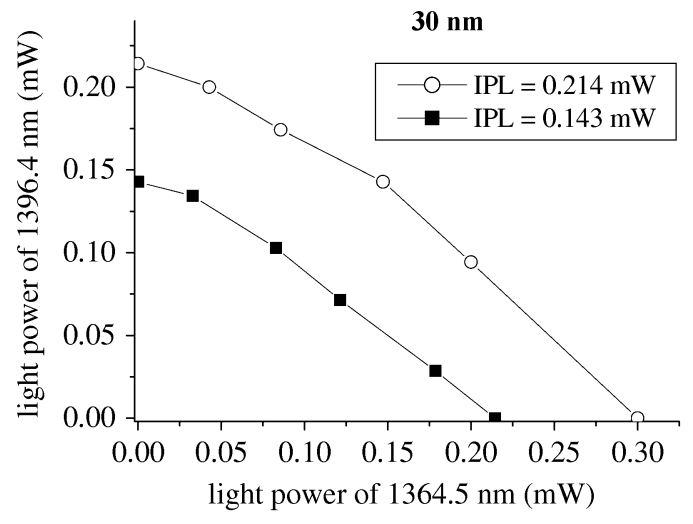

(e)

Fig. 2. Experimental curves of LWM power to SWM power for different wavelength separation: (a) $170 \mathrm{~nm}$. (b) $150 \mathrm{~nm}$. (c) $130 \mathrm{~nm}$. (d) $60 \mathrm{~nm}$. (e) $30 \mathrm{~nm}$. The wavelength position of the SWM is fixed to around $1360 \mathrm{~nm}$. The initial power of the LWM (IPL) for each curve is listed in the figures.

causes the LWM power to increase from 0.07 to $0.21 \mathrm{~mW}$, which is only a $0.14-\mathrm{mW}$ increment. However, at $170-\mathrm{nm}$ separation, anticompetition causes the LWM power to increase from 0.07 to $0.3 \mathrm{~mW}$, which is a $0.23-\mathrm{mW}$ increment.

The experimental results shown in Fig. 2 are done with the fixed wavelength of the SWM. The experiments are also done with a fixed wavelength of the LWM. In this experiment, the LWM is fixed at about $1528 \mathrm{~nm}$, while the SWM moves toward the long-wavelength side when we change the wavelength separation. The resulting curves are not exactly the same as those shown in Fig. 2 and will be discussed in Section III-D. However, the trend is the same. That is, both the maximum LWM power increment and the gradient of the anticompetition curve under fixed initial LWM power increase when we increase the wavelength separation. Also, anticompetition can hardly be observed at wavelength separation smaller than $60 \mathrm{~nm}$.

The reason for not observing anticompetition at wavelength separation below $60 \mathrm{~nm}$ is as follows. Anticompetition is caused by the mechanism similar to optical pumping [17]. When the SWM and LWM are closely separated, the difference of photon energy between them is very small. Therefore, the mechanism of optical pumping is very weak, leading to negligible anticompetition.

On the other hand, when the two wavelengths are widely separated, their gains significantly differ. There may be a situation where the gain of the LWM is near the lasing threshold while 


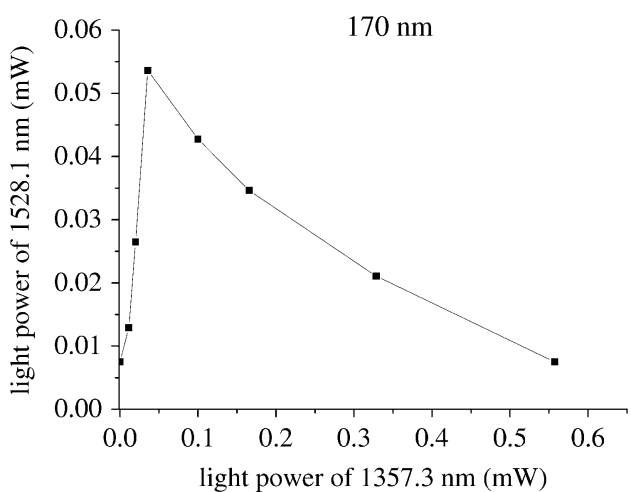

Fig. 3. Experimental curves of $170-\mathrm{nm}$ separation. The initial power of the LWM is close to zero.

that of the SWM is highly above the threshold. Then the LWM hardly oscillates and, thus, mode competition does not occur. However, due to optical pumping, the oscillating SWM can provide the LWM with some optical gain. If the initial LWM gain plus the extra optical gain is larger than the LWM threshold, the LWM will start oscillation, giving rise to the anticompetition. To verify this inference, we do another experiment. This experiment consists of two steps. First, the loss of the long-wavelength ND filter is increased so that the lasing peak of the LWM on the spectrum is very weak, i.e., only slightly larger than the background noise. Then, we gradually lower the loss of the short-wavelength ND filter to obtain the experimental curve. Fig. 3 shows the experimental result for the wavelength separation $170 \mathrm{~nm}$. From Fig. 3, we see that anticompetition does elevate the LWM power from about zero to more than $0.05 \mathrm{~mW}$. This experimental result verifies that at large wavelength separation, anticompetition can help the LWM to oscillate if initially the LWM has insufficient gain for oscillation.

Increasing wavelength separation will not only increase the gradient of the anticompetition curve, but also decrease the absolute value of the competition curve gradient. From Fig. 2, we can see that at $150-\mathrm{nm}$ separation, the competition curves are not as steep as those at 130- and 60-nm separations. In addition, at $170-\mathrm{nm}$ separation, the gradient of the competition curve is close to zero. This is because at large wavelength separation, most of the carriers are contributed from different QWs. Compared to the intraband relaxation in the same well, carrier transportation between different QWs is a relatively slow process. Therefore, competition at large wavelength separation is weak. In addition, the anticompetition mechanism further weakens the competition process.

It is worth noting that the competition curves in Fig. 2(e) are not straight lines. They are curves with downward curvature. The "less negative" gradient appearing in the low SWM power region somewhat indicates that there is still the mechanism of anticompetition at 30-nm separation. However, compared with competition, the influence of anticompetition is very weak. Therefore, the behavior of the system is dominated by competition.

\section{B. Power of the SWM}

Anticompetition exists only when the power of the SWM is below a certain level. For example, in Fig. 2(a), the anticom- petition section can exist only when the power of the SWM is below $0.07 \mathrm{~mW}$. If the power of the SWM is increased to above $0.07 \mathrm{~mW}$, anticompetition disappears, and the interaction between these two modes gradually turns into competition. In general, increasing the wavelength separation will also increase the existing range of anticompetition.

Since mode competition dominates the behavior of the laser system when the SWM power is above a certain level, LWM will be completely suppressed by the SWM if the SWM power is large enough. However, due to the loss of the external cavity, sometimes the SWM power cannot reach such a large value that the LWM is completely suppressed. Therefore, some curves in Fig. 2 do not end at the point where the LWM power is zero. This phenomenon is most likely to occur at the large wavelength separation because the SWM cannot reach a sufficiently large power.

\section{Initial Power of the LWM}

The situation of anticompetition is also influenced by the initial power of the LWM. The experiments show that, if the initial power of the LWM decreases, both the gradient of the anticompetition curve and the maximum LWM power increment increase. For example, in Fig. 2(a), if the initial LWM power is about $0.27 \mathrm{~mW}$, anticompetition can elevate the LWM power to about $0.37 \mathrm{~mW}$, which is only a $0.1-\mathrm{mW}$ increment. However, if the initial LWM power is only about $0.07 \mathrm{~mW}$, anticompetition can result in a LWM power increment larger than $0.23 \mathrm{~mW}$. The same phenomenon can also be observed in Fig. 2(b) and (c).

Why can the initial LWM power affect the LWM power increment caused by anticompetition? The reason is as follows. Because the injection current is fixed to $146 \mathrm{~mA}$, the total gain of the laser system is constant. Therefore, if the initial LWM power is large, the LWM has already taken up a large portion of the system gain. Then the available SWM gain will become small. In this situation, anticompetition is weak because there is not enough SWM gain to provide a function like optical pumping. In fact, when the initial LWM power is very large, the SWM will cease oscillation even if we remove the short-wavelength ND filter. When the initial LWM power is low, the power of the SWM can be large enough to provide gain for optical pumping, so anticompetition is strong.

Fig. 2 also shows that decreasing the initial LWM power can result in decreasing maximum achievable LWM power although the corresponding LWM power increment is large. The low initial LWM power corresponds to a high cavity loss, which can be achieved by adding the loss via the long-wavelength ND filter. In this case, even when the SWM provides it some optical gain, its maximum achievable LWM power cannot be as strong. On the other hand, the relatively weak maximum achievable LWM power causes it to have weak competition ability against the SWM. Then SWM begins to rob the available gain of LWM at a reduced power level. As a result, the low initial LWM power also reduces the existing range of anticompetition, as shown in Fig. 2(a)-(c).

The phenomenon that the maximum achievable LWM power decreases with the initial LWM power as discussed above is more apparent under small wavelength separation than under large wavelength separation. For example, consider the 170-nm 


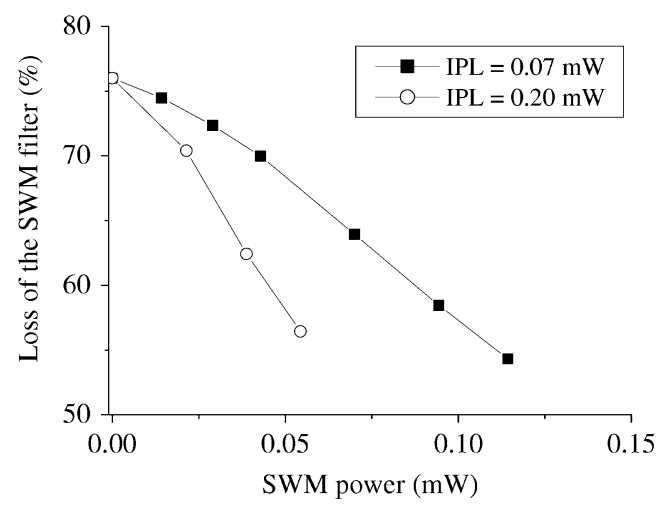

Fig. 4. Loss introduced by the SWM filter at different SWM power. The wavelength positions are the same as in Fig. 2(b). The initial power of the LWM (IPL) is listed in the figure.

separation shown in Fig. 2(a). At initial LWM power 0.07 and $0.13 \mathrm{~mW}$, the maximum LWM power is 0.31 and $0.325 \mathrm{~mW}$, respectively. Therefore, for these two initial LWM powers, the difference of the maximum LWM power equals $0.015 \mathrm{~mW}$. For 130-nm separation shown in Fig. 2(c), again, at initial LWM power 0.07 and $0.13 \mathrm{~mW}$, the maximum LWM power is 0.12 and $0.182 \mathrm{~mW}$, respectively, so the difference of the maximum LWM power is $0.062 \mathrm{~mW}$. Obviously, the decrease in maximum achievable LWM power is larger at $130-\mathrm{nm}$ separation than at $170-n m$ separation. The reason is as follows. As discussed above, the LWM has weak competition ability against the SWM when the initial LWM power is small. Then the SWM begins to rob the available gain of LWM at a reduced power level, which results in the phenomenon that the maximum achievable LWM power decreases with the initial LWM power. However, at large wavelength separation, competition is weak even at low initial LWM power. Therefore, at large wavelength separation, the LWM can still sustain the competition from the SWM even if the initial LWM power is low. On the other hand, competition is strong at small wavelength separation. If the loss of the LWM is large, the SWM can easily rob the available gain of the LWM and results in a significant reduction in the maximum achievable LWM power. Consequently, with the same amount of decrease in the initial LWM power, the decrease in the maximum LWM power is larger at small separation than at large separation.

In Fig. 2, under fixed SWM power, the LWM power is not the same at different initial LWM power. It seems that the total power is not constant under fixed injection current. However, it should be noted that the horizontal axis in Fig. 2 is the SWM power, not the loss introduced to the SWM by the ND filter. Fig. 4 shows the filter loss at different SWM power for the curves with initial LWM power 0.07 and $0.2 \mathrm{~mW}$ in Fig. 2(b). It shows that to obtain the same SWM power, the loss of the short-wavelength ND filter has to decrease with increasing initial LWM power. The smaller external loss means larger available gain under the fixed injection current, leading to larger power levels. Therefore, the total power of different curves does not have to be the same.

\section{Wavelength Position}

Different wavelength position could result in different behaviors of anticompetition even at fixed wavelength separation.
The experiment goes through as follows. First, we move the double-slit vertically to choose certain wavelength separation, and then fix this separation. Second, we move the double-slit horizontally to shift the wavelength position of the SWM and LWM at the same time. The experimental results for wavelength separation 150 and $130 \mathrm{~nm}$ are shown in Fig. 5. In Fig. 5(a) and (c), the SWM is located at about $1360 \mathrm{~nm}$. Both the SWM and LWM in Fig. 5(b) and (d) are shifted toward the long-wavelength side with respect to Fig. 5(a) and (c), respectively. The LWM of Fig. 5(b) and (d) is located at about $1527 \mathrm{~nm}$. Comparing Fig. 5(a) with (b), we find that with fixed initial LWM power, the gradient of the anticompetition curve is larger in Fig. 5(a), and the maximum LWM increment is also larger in (a). That is, at wavelength separation $150 \mathrm{~nm}$, both the maximum LWM power increment and the gradient of the anticompetition curve increase with decreasing wavelength of both modes. At wavelength separation $130 \mathrm{~nm}$, the gradient of the anticompetition curve also increases with decreasing wavelength of both modes, but the maximum LWM power increment decreases with decreasing wavelength of both modes, as shown in Fig. 5(c) and (d).

To realize the influence of the wavelength position on anticompetition, we have to consider the gain curve of the laser gain medium. For the gain medium used in the experiment, the $\mathrm{In}_{0.67} \mathrm{Ga}_{0.33} \mathrm{As}_{0.72} \mathrm{P}_{0.28} \mathrm{QW}$ has its first quantized state located in $1300 \mathrm{~nm}$, while the $\mathrm{In}_{0.53} \mathrm{Ga}_{0.47} \mathrm{As} \mathrm{QW}$ has its first and second quantized states located in 1540 and $1370 \mathrm{~nm}$, respectively. Since the wavelength of the SWM is at least $60 \mathrm{~nm}$ away from the first quantized state of the $\mathrm{In}_{0.67} \mathrm{Ga}_{0.33} \mathrm{As}_{0.72} \mathrm{P}_{0.28}$ $\mathrm{QW}$, most of the carriers contributing to this mode are supported by the $\mathrm{In}_{0.53} \mathrm{Ga}_{0.47} \mathrm{As} \mathrm{QW}$ s that contribute to emission above $1300 \mathrm{~nm}$. Therefore, the gain curve of the laser gain medium can be approximated by the gain curve of the $\operatorname{In}_{0.53} \mathrm{Ga}_{0.47} \mathrm{As}$ QW. The gain curve of the $\operatorname{In}_{0.53} \mathrm{Ga}_{0.47}$ As QW can be theoretically calculated [22] or experimentally obtained with the following procedure. First, we remove the variable ND filters and change the double slits into a single slit. Then, we shift the slit to tune the lasing wavelength from 1360 to $1540 \mathrm{~nm}$. Each time the slit is shifted to a new place, the alignment of the external cavity is optimized to obtain the maximum achievable power at that wavelength. After finishing this experiment, we can obtain a curve that represents the relation between the maximum achievable power and wavelength, as shown in Fig. 6. Although the curve shown in Fig. 6 is not the actual gain curve, its profile approximates the gain curve and is not much different from the calculated result using the model given by [22]. The curve in Fig. 6 is of bell shape, with a peak located at about $1470 \mathrm{~nm}$. There is a dip around $1395 \mathrm{~nm}$. Because the overall gain profile is the overlap of gains contributed from two different types of QWs [18], the dip is where both types of QWs give relatively small contribution to the gain. This dip shows no influences on the anticompetition.

The reason that wavelength position can affect the slope of the anticompetition curve and the maximum LWM power increment is as follows. The slope of the anticompetition curve is the LWM power increment divided by the SWM power increment. Therefore, this slope tells us qualitatively how much SWM power can be transferred successfully into the 


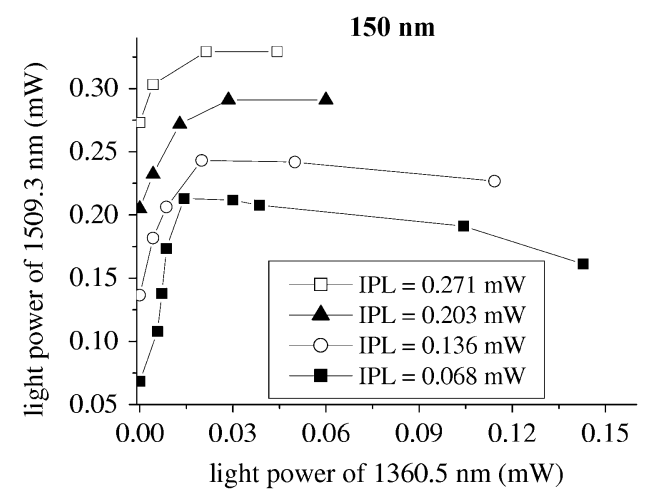

(a)

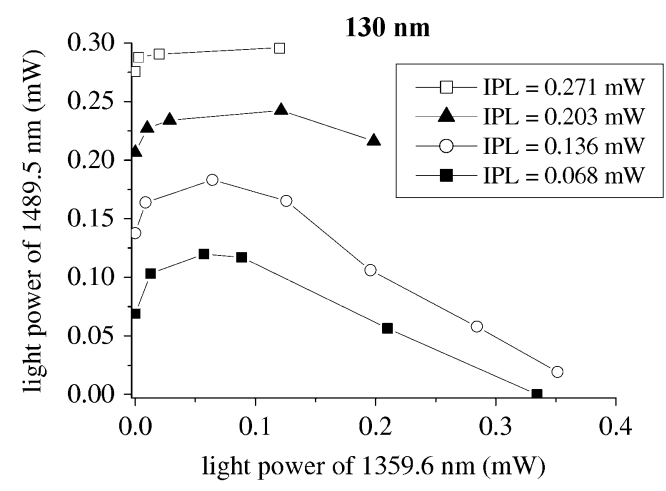

(c)

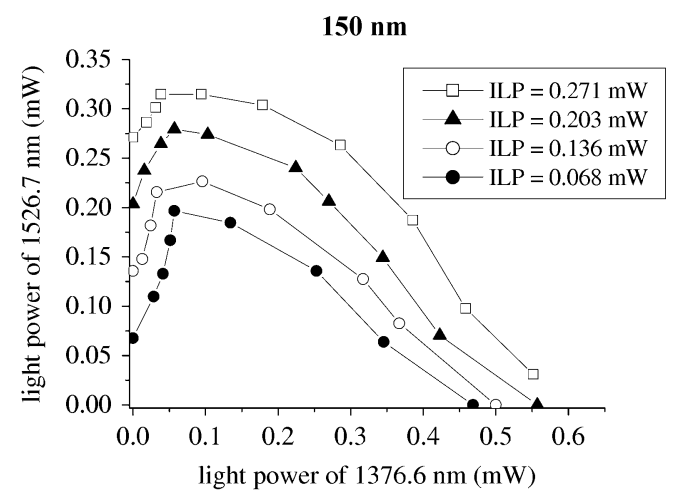

(b)

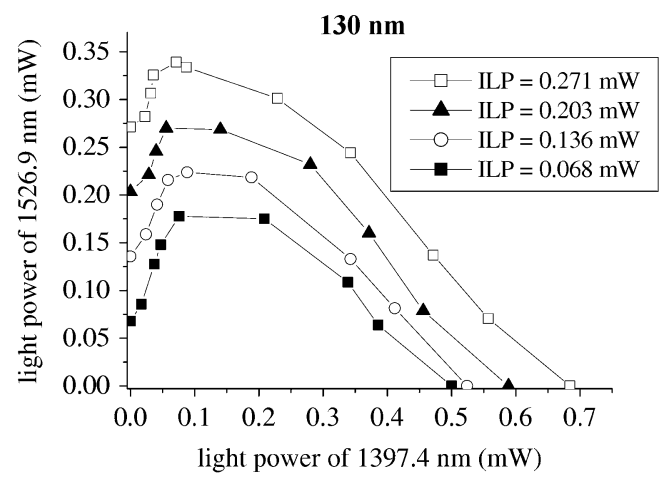

(d)

Fig. 5. Experimental curves for different wavelength position with fixed wavelength separation (WS): (a) $W S=150 \mathrm{~nm}$. (b) $W S=150 \mathrm{~nm}$., (c) $W S=130$ nm. (d) $W S=130 \mathrm{~nm}$. The initial power of the LWM (IPL) is listed in the figure.

LWM power. Large slope indicates that small SWM power is needed to obtain certain LWM power. The function of optical pumping can be divided into two steps. First, the SWM photon is absorbed by high energy states. Second, those carriers relax to the energy states corresponding to the long wavelength. The absorbed photons thus increase the population inversion in the long-wavelength states, and so increase the oscillation intensity of the LWM. Therefore, the power transfer from the SWM to the LWM is determined by two terms. The first one is the photon absorption ability. The second one is the transferring ability from the population inversion to the LWM power. If the photon absorption ability of the long-wavelength QW is assumed to be constant, the power transfer from the SWM to the LWM is determined by the transferring ability from the population inversion to the LWM power.

If an oscillation mode is located at the wavelength with a small gain, only a small amount of the population inversion can contribute to that particular mode no matter if the gain is provided by the injected current or by optical absorption. Therefore, if the LWM is located in the low gain region, the power transfer from the SWM to the LWM is weak, and thus the slope of the anticompetition curve is small. From Fig. 6, we can find that the LWM is located at the wavelength with a higher gain in Fig. 5(a) than in Fig. 5(b). This can also be verified from the emission spectra in [18] for the operation current below $400 \mathrm{~mA}$. Therefore, the power transfer from the SWM to the LWM in Fig. 5(a) is stronger than that in Fig. 5(b). Consequently, the slope of the anticompetition curve is larger in Fig. 5(a) than in Fig. 5(b).

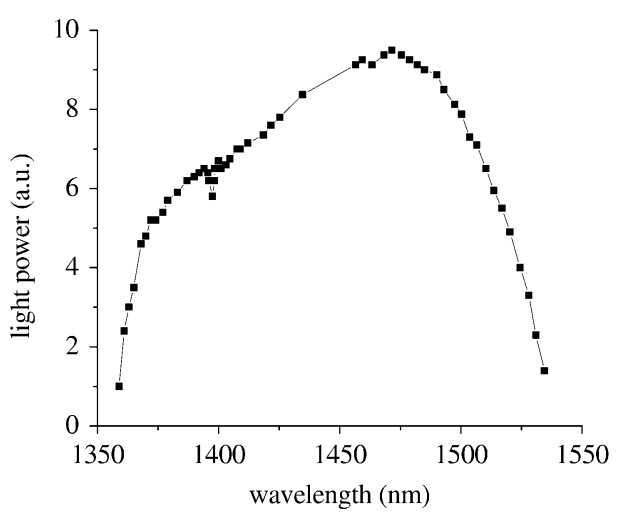

Fig. 6. Variation of maximum available optical power to wavelength position. This curve is used as an approximation to the gain curve of the $\operatorname{In}_{0.53} \mathrm{Ga}_{0.47} \mathrm{As}$ QW.

Similarly, the slope of the anticompetition curve is also larger in Fig. 5(c) than in Fig. 5(d) because the LWM in Fig. 5(c) is located at the wavelength with a higher gain.

The wavelength position can also affect the maximum LWM power increment. The reason is as follows. Because the LWM power increment comes from the SWM power, only SWM with large gain can offer large increment in LWM power. On the other hand, the slope of the anticompetition curve can also affect the LWM power increment. If the gain variation of the SWM is small, the slope of the anticompetition curve will dominate the behavior of the maximum LWM power increment at different 
wavelength position. For example, consider Figs. 5 and 6. Although the gain of the SWM in Fig. 5(a) is smaller than that in Fig. 5(b), the maximum LWM power increment is still larger in Fig. 5(a) than in Fig. 5(b) under a fixed initial LWM power. This is because their SWM gains do not differ very much and that the slope of the anticompetition curve is larger in Fig. 5(a). Since a larger slope of the anticompetition curve implies more SWM power being successfully transferred into the LWM power, it is reasonable that the maximum LWM power increment is larger in Fig. 5(a) than in Fig. 5(b). On the contrary, the maximum LWM power increment is larger in Fig. 5(d) than in Fig. 5(c) under a fixed initial LWM power. This is because the SWM gain in Fig. 5(d) is much larger than that in Fig. 5(c), so the maximum LWM power increment is dominated by the SWM gain.

\section{E. Injection Current}

The experimental results demonstrated above are all obtained under 146-mA injection current. We have also done experiment with the injection current larger than $146 \mathrm{~mA}$. In general, increasing the injection current has two effects. First, it elevates the total gain and leads to a blue shift of the lasing wavelength due to the band-filling effect. Second, it results in an increase in temperature. The increased temperature usually causes the lasing wavelength to be red-shifted for normal QW lasers because the bandgap energy is reduced. However, for semiconductor lasers with nonidentical MQWs, the temperature increase may lead to the increase of the gain for the SWM and the decrease of the gain for the LWM [20]. The reason is due to the strongly temperature-dependent Fermi-Dirac distribution, which favors carriers in high-energy states at large temperature [20]. Therefore, for the semiconductor lasers with nonidentical MQWs in this case, the gain of the SWM always increases with the injection current. On the other hand, the gain of the LWM may decrease with increasing injection current as a result of the temperature effect, depending on the wavelength position of the LWM and the injection current level. Therefore, when we increase the injection current, the gain increment of the SWM is larger than that of the LWM because the increased carriers are more likely captured by the short-wavelength QWs.

Fig. 7 show the experimental results under injection current 146 and $149 \mathrm{~mA}$ for different wavelength separation. In Fig. 7(a), the wavelength separation is $150 \mathrm{~nm}$. It is clear that both the maximum LWM power increment and the slope of the anticompetition curve increase with increasing injection current. Because the gain of the SWM increases at the increased injection current, the SWM can offer more gain to increase the increment of the LWM power. In addition, since the gain of the LWM also increases when the injection current increases from 146 to $149 \mathrm{~mA}$, the slope of the anticompetition curve increases with the injection current. This is because the energy transfer form the SWM to the LWM is efficient when the LWM gain is large, as discussed in Section III-D.

The competition curve in Fig. 7(a) also has its slope "increasing" with the injection current. The reason is as follows. As discussed above, when we increase the injection current, the gain increase of the SWM is always larger than that of the LWM. Therefore, under high injection current, the SWM is more competitive than the LWM. As a result, the slope of the competition

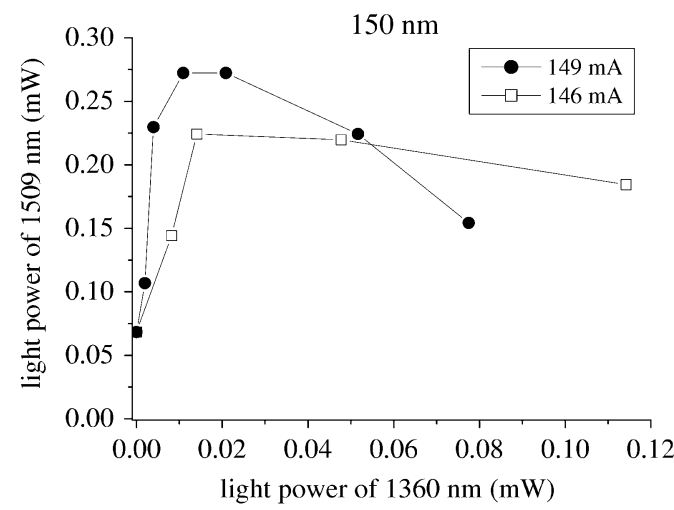

(a)

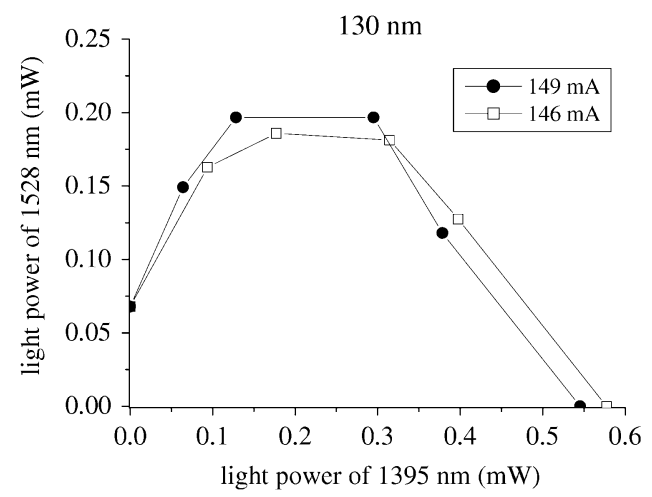

(b)

Fig. 7. Relation between the SWM and LWM power under different injection current. (a) 150-nm separation. (b) 130-nm separation.

curve "increases" with increasing injection current when the experiment is done with the varying SWM power.

At the 130-nm separation shown in Fig. 7(b), the behaviors of anticompetition do not differ very much for different injection current levels. However, both competition and anticompetition are still slightly more sever for the injection current 149 than $146 \mathrm{~mA}$.

\section{VARYing Long-WaVelength Mode Power}

The above experiments are done with fixed loss of the long-wavelength ND filter. The loss of the short-wavelength ND filter is varied to change the power of the SWM and the power of the LWM changes accordingly. We have also done another experiment. The loss of the short-wavelength ND filter is fixed, but the loss of the long-wavelength ND filter is varied to change the power of the LWM. The power of the SWM then also varies accordingly. Fig. 8 shows the experimental results for wavelength separation $170 \mathrm{~nm}$. The wavelength positions of the SWM and LWM in Fig. 8 are the same as that in Fig. 2(a). In Fig. 8, we do not observe anticompetition. That is, the power of the SWM always decreases when we increase the power of the LWM. Similar behaviors are also obtained for different wavelength separations. The reason for only competition existing in varying LWM power is because anticompetition is caused by the mechanism similar to optical pumping and the photon energy of the LWM is smaller than that of the SWM. Therefore, the LWM photon cannot provide the SWM with optical gain, and thus anticompetition cannot occur. 


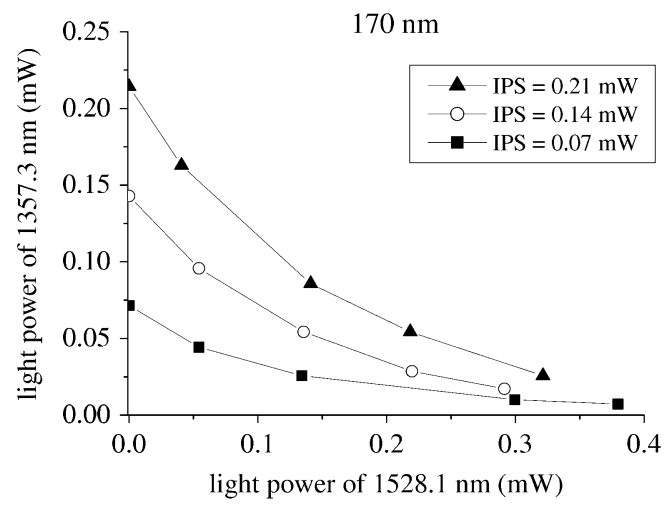

Fig. 8. Experimental curves for varying the power of the LWM. The initial power of the SWM (IPS) is listed in the figure.

\section{CONCLUSION}

The behaviors of anticompetition are investigated under different operation conditions. Anticompetition does not dominate in the material with two different types of QWs in our investigation when the wavelength separation is larger than $60 \mathrm{~nm}$. In addition, anticompetition will vanish when the power of the SWM reaches a certain power level. At large wavelength separation or under small initial power of the LWM, both the slope of the anticompetition curve and the maximum power increment of the LWM are large. Different wavelength position also result in different behaviors of anticompetition even under a fixed wavelength separation. Finally, both competition and anticompetition are severe under high injection current. The anticompetition cannot be observed when we change the power of the LWM.

\section{REFERENCES}

[1] W. E. Lamb, "Theory of an optical maser," Phys. Rev. A, Gen. Phys., vol. 134, pp. A1429-A1450, 1964

[2] A. E. Siegman, Lasers. Mill Valley, CA: Univ. Sci. Books, 1986, pp. 992-999.

[3] M. Kuznetsov, "Picosecond switching dynamics of a bistable-wavelength-latch two-segment distributed feedback laser," IEEE Photon. Technol. Lett., vol. 2, no. 3, pp. 623-625, Mar. 1990.

[4] K.-Y. Liou and C. A. Burrus, "Electro-optical logic operations with twoelectrode distributed feedback injection lasers," Appl. Phys. Lett., vol. 51, pp. 1777-1779, 1987.

[5] C.-F. Lin and P.-C. Ku, "Analysis of stability in two-mode laser systems," IEEE J. Quantum Electron., vol. 32, no. 8, pp. 1377-1382, Aug. 1996.

[6] M. Watanabe, H. Itoh, S. Mukai, and H. Yajima, "Optical tristability using a twin-stripe laser diode," Appl. Phys. Lett., vol. 50, pp. 427-429, 1987.

[7] S. Noda, Y. Kobayashi, K. Shibata, and A. Sasaki, "Optoelectronic integrated tristable device with optically controlled set and reset functions," IEEE J. Quantum Electron., vol. 31, no. 8, pp. 1465-1473, Aug. 1995.

[8] S. Sivaprakasam, P. S. Spencer, P. Rees, and K. A. Shore, "Regimes of chaotic synchronization in external-cavity laser diodes," IEEE $J$. Quantum Electron., vol. 38, no. 9, pp. 1155-1161, Sep. 2002.

[9] H. Kawaguchi, Bistability and Nonlinearities in Laser Diodes. Norwood, MA: Artech House, 1994, p. 199.

[10] H. Kawaguchi and I. S. Hidayat, "Gigahertz all-optical flip-flop operation of polarization-bistable vertical-cavity surface emitting lasers," Electron. Lett., vol. 31, pp. 1150-1151, 1995.

[11] G. D. VanWiggeren and R. Roy, "Communication with chaotic lasers," Science, vol. 279, pp. 1198-1200, 1998.
[12] X. Jin and S. L. Chuang, "Relative intensity noise characteristics of injection-locked semiconductor lasers," Appl. Phys. Lett., vol. 77, pp. $1250-1252,2000$

[13] H. Statz and C. L. Tang, "Phase-locking of modes in lasers," J. Appl. Phys., vol. 36, pp. 3923-3927, 1965.

[14] L. E. Hargrove, R. L. Fork, and M. A. Pollack, "Locking of He-Ne laser modes induced by synchronous intracavity modulation," Appl. Phys. Lett., vol. 5, pp. 4-5, 1964.

[15] P. W. Smith, "Mode-locking of lasers," Proc. IEEE, vol. 58, pp. 1342-1357, Sep. 1970.

[16] I. White, R. Penty, M. Webster, Y. J. Chai, A. Wonfor, and S. Shahkooh, "Wavelength switching components for future photonic networks," IEEE Commun. Mag., vol. 40, no. 9, pp. 74-81, Sep. 2002.

[17] C.-F. Lin, C.-C. Huang, F.-H. Chu, and Y.-S. Su, "Anticompetition of laser modes," Appl. Phys. Lett., vol. 82, pp. 3611-3613, 2003.

[18] C.-F. Lin, B.-R. Wu, L.-W. Laih, and T.-T. Shih, "Sequence influence of nonidentical InGaAsP quantum wells on broad-band characteristics of semiconductor optical amplifiers-superluminescent diodes," Opt. Lett., vol. 26, pp. 1099-1101, 2001.

[19] C.-F. Lin, Y.-S. Su, and B.-R. Wu, "External-cavity semiconductor laser tunable from 1.3 to $1.54 \mu \mathrm{m}$ for optical communication," IEEE Photon. Technol. Lett., vol. 14, no. 1, pp. 3-5, Jan. 2002.

[20] C.-F. Lin, Y.-S. Su, D.-K. Yu, C.-H. Wu, and B.-R. Wu, "Improved temperature characteristics of laser diodes with nonidentical multiple quantum wells due to temperature-Induced carrier redistribution," Appl. Phys. Lett., vol. 82, pp. 3403-3405, 2003.

[21] C.-C. Huang, C.-H. Cheng, Y.-S. Su, and C.-F. Lin, "174 nm mode spacing in dual-wavelength semiconductor laser using nonidentical InGaAsP quantum wells," IEEE Photon. Technol. Lett., vol. 16, no. 2, pp. 371-373, Feb. 2004.

[22] C.-S. Chang and S. L. Chuang, "Modeling of strained quantum-well lasers with spin-orbit coupling," IEEE J. Select.Topics Quantum Electron., vol. 1, no. 2, pp. 218-229, Jun. 1995.

Chi-Chia Huang was born in Taipei, Taiwan, R.O.C., in 1980. He received the B.S. degree in electrical engineering and M.S. degree in electrooptical engineering from National Taiwan University, Taipei, Taiwan, R.O.C., in 2002 and 2004, respectively.

His research interest is on semiconductor lasers and amplifiers.

Yi-Shin Su was born in Chiayi City, Taiwan, R.O.C., in 1978. He received the B.S. degree in electrical engineering and the M.S. degree in electrooptical engineering in 2000 and 2002, respectively, from National Taiwan University, Taipei, Taiwan, R.O.C., where he is currently working toward the Ph.D. degree.

His current research interests include broad-band SOAs based on nonidentical MQW structures, high-power diode lasers, and mode-locked diode lasers.

Ching-Fuh Lin (S'89-M'92-SM'00) was born in I-Lan, Taiwan, R.O.C., in 1961. He received the B.S. degree from National Taiwan University, Taipei, and the M.S. and Ph.D. degrees from Cornell University, Ithaca, NY, in 1989 and 1993, respectively, all in electrical engineering.

In 1993, he joined the faculty of National Taiwan University in the Department of Electrical Engineering and the Graduate Institute of Electro-Optical Engineering, then also the Graduate Institute of Electronics Engineering. His current research interests include broad-band semiconductor lasers/amplifiers, high-power semiconductor lasers, light emission on/from $\mathrm{Si}$, and nanostructures/physics for optoelectronics.

Prof. Lin is a Senior Member of the IEEE Lasers and Electro-Optics Society and a member of the Optical Society of America and SPIE. 